\title{
A fast visual colorimeter
}

\author{
CHARLES W. WHITE \\ Duke University, Durham, North Carolina 27706 \\ MYRON L. WOLBARSHT \\ Department of Ophthalmology, Duke Medical Center, Durham, North Carolina 27710 \\ and \\ TODD TIEGER \\ Stanford University, Stanford, California 94305
}

\begin{abstract}
A simple colorimeter is described which enables untrained observers to rapidly and reliably match hue and saturation using a novel joystick control. The colorimeter output is suitable for computer recording and analysis. The applications of this color-matching technique in experiments requiring speed and simplicity are illustrated.
\end{abstract}

Many visual colorimeters have two kinds of drawbacks for research in color vision. They tend to be large, cumbersome, and expensive instruments, and they often require complicated adjustments in order to make color matches. Most colorimeters, for example, require independent adjustment of the intensity controls of from three to six primary light sources (Wyszecki \& Stiles, 1967). A simpler device would be useful when the observers are inexperienced, when changing adaptation makes fast matches necessary, or when time and space constraints in the experimental environment preclude long colormatching sessions.

For many research purposes in the psychology of color vision, inexperienced subjects are preferred over the more traditional trained observers. But naive subjects often cannot quickly learn to use complicated colorimeters. Such subjects already know, or can learn very quickly, the concept of a color surface with the hue circle around the perimeter, neutral colors in the center, and saturation increasing toward the rim of the circle. A simple colored picture is often sufficient instruction. We can simplify the task of color matching for some subjects by introducing opponent-color principles and by describing two orthogonal dimensions of the color surface as a

We thank Mike Bessler and David Montgomery for their help in conducting the experiments and George Carver for his assistance in constructing the colorimeter. The research was supported by a Duke University Research Council grant to Charles W. White and by N.A.S.A. Contract NAS 9-11994 with Myron L. Wolbarsht. Address correspondence to Charles W. White, Department of Psychology, Duke University, Durham, North Carolina 27706. red-green axis and a yellow-blue axis. A colorimeter designed to work along those lines facilitates color matching for many observers. We have recently used such a colorimeter successfully to obtain colormatching data from inexperienced subjects. After describing the apparatus, we illustrate its application with results from two experiments.

\section{APPARATUS}

The basic colorimeter is a modification of one used by Festinger, Allyn, and White (1971), which was first described by Burnham (1952). Wyszecki and Stiles (1967) describe the principles of the device, and Wright (1969) illustrates a compact model of Burnham's design. Our version consists of an X-Y plotter (BBN Plotamatic Model 715), three Wratten filters, an optical integrating bar (described below), a 150 -W xenon arc lamp (Osram XBO $150 \mathrm{~W}$ in Leitz housing), and a joystick control (Digital Equipment Corporation, Model H306) interfaced with a PDP-8/L computer.

Figure 1 is a plan view of the components. The arc lamp's high-intensity white light is collimated by an achromatic lens and projected through the filter slide onto the surface of the integrating bar. The integrating bar is composed of two $36 \times 36 \mathrm{~mm}$ diffusing plates of tlashed opal glass cemented to the ends of a clear acrylic bar, $36 \times 36 \times 90 \mathrm{~mm}$. The diffuse light which penetrates the first surface undergoes multiple internal reflections within the bar and is again diffused by the second opal glass surface, 


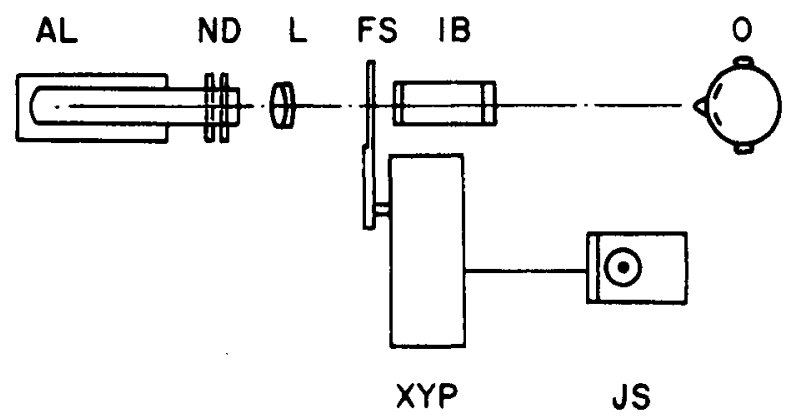

Figure 1. A schematic top view of the colorimeter apparatus. Symbols are as follows: AL, arc lamp; ND, neutral density filters; L, achromatic lens; FS, filter slide; IB, optical integrating bar; O, observer; XYP, X-Y plotter; JS, joystick control. Not drawn to scale. See text for details.

which produces a uniformly mixed color field. The filter slide depicted in Figure 2 is an $80 \times 80 \mathrm{~mm}$ square of three Wratten filters mounted between glass covers and rigidly connected by a clear acrylic frame to the pen holder of the plotter. The filters are arranged with the lower half blue (Wratten No. 47B), the upper left quadrant red (Wratten No. 70), and the upper right quadrant green (Wratten No. 74), as viewed from the direction of the integrating bar.

The joystick control contains two potentiometers mounted perpendicularly and gimbaled to measure the position of a hand-held control lever. Moving the joystick changes the voltage set by each potentiometer between -1.5 and $+1.5 \mathrm{~V}$. These voltage signals drive the plotter directly to determine the color mixture. The joystick signals also go to an analog-to-digital converter in the computer's oscilloscope control unit (Digital Equipment Corporation KV8 graphics system). We programmed the computer to read the two joystick voltages when the observer pressed a microswitch at the base of the joystick. The computer then converted the analog voltages to CIE chromaticity coordinates according to the equations described in the calibration procedure, stored the data for later statistical analysis, and printed the results. Although the on-line computer made the conversions quickly and simply. any two independent voltage outputs are sufficient to operate the device. The joystick itself can be replaced by two 10-turn potentiometer sources and the voltages conveniently monitored by digital voltmeters (Wolbarsht, White, \& Anderson, Note 1).

\section{CALIBRATION PROCEDURE}

The following steps were carried out to calibrate the movements of the plotting arm with that of the joystick. The position of the joystick handle was adjusted to give zero voltage readings on both dimensions. Then we moved the filter slide to a position directly over the center point of the mixing cube surface by adjusting the plotter's zero-balance controls. Next, we moved the joystick to its extreme positions to achieve the maximum positive or negative voltage $( \pm 1.5 \mathrm{~V})$. Finally, we adjusted the plotter's amplitier gain controls so that the outer corners of the filter slide coincided with the corners of the mixing cube surface.

Calculation of the percentages of each filter's contribution to the light mixture on the mixing cube surface was done by algebraically shifting the zero point and normalizing the interval such that the joystick readings varied from 0 to 1 . The computer interpreted a joystick voltage as an integer between -512 and +512 . We used only the range between \pm 450 and rescaled the values by adding 450 and dividing by 900 , which gave filter slide coordinates as fractions of the total travel horizontally (h) or vertically (v). Given the arrangement of filters shown in Figure 2, a filter position $(\mathrm{h}, \mathrm{v})$ was transformed into the relative contribution of each filter in the color match by the following equations:

$$
\begin{aligned}
& \mathrm{p}_{\mathrm{r}}=\mathrm{v}(\mathrm{l}-\mathrm{h}) \\
& \mathrm{p}_{\mathrm{g}}=\mathrm{h} \mathrm{v} \\
& \mathrm{p}_{\mathrm{b}}=1-\mathrm{v}
\end{aligned}
$$

Energy spectra were measured for the combination of light source, lens, mixing bar, and each filter with a spectroradiometer (Instrumentation Specialities Company, Model SR) and fiber optics probe attachment placed against the second opal glass surface, the exit surface. We measured energy at $10-\mathrm{nm}$ wavelength intervals across the visible spectrum, taking into account the manufacturer's wavelength compensation curve for the probe and spectroradiometer combination. The tristimulus values for the $1931 \mathrm{CIE}$ standard observer we obtained are indicated in Table 1.

The CIE tristimulus values for any mixture $\left(\mathrm{X}_{\mathrm{m}}\right.$,

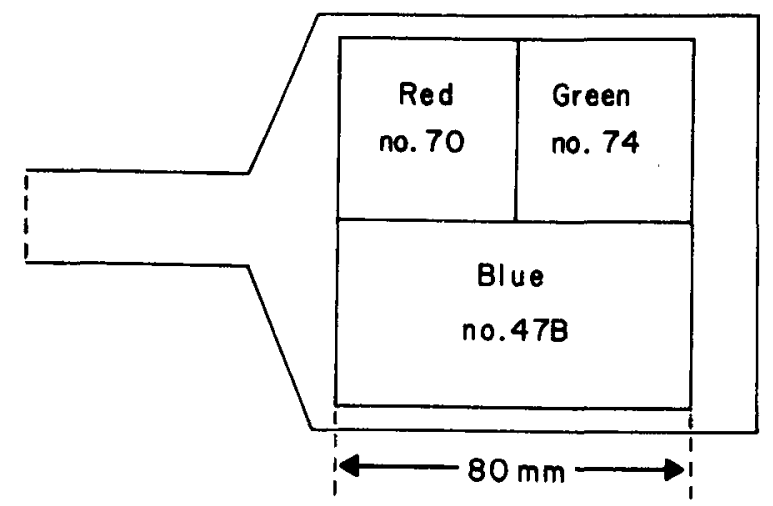

Figure 2. The filter slide viewed from the mixing cube side. The three filters, labeled in the diagram by Wratten filter numbers, were mounted between cover glasses in an acrylic frame and attached to the X-Y plotter arm. 
Table 1

CIE Tristimulus Values of Filter Primaries

Tristimulus Values

\begin{tabular}{lrrr} 
& \multicolumn{3}{c}{ Tristimulus Values } \\
\cline { 2 - 4 } Filter & \multicolumn{1}{c}{$\mathrm{X}$} & $\mathrm{Y}$ & \multicolumn{1}{c}{$\mathrm{Z}$} \\
\hline Red $(70)^{*}$ & 22.990 & 9.190 & .100 \\
Green (74)* & 3.432 & 13.047 & 3.398 \\
Blue (47B)* & 8.658 & 16.629 & 39.430 \\
\hline
\end{tabular}

*Wratten filter numbers are in parentheses after the nominal colors.

$\mathrm{Y}_{\mathrm{m}}$, and $\mathrm{Z}_{\mathrm{m}}$ ) are calculated according to Equations 2, which weight the $\mathrm{X}, \mathrm{Y}, \mathrm{Z}$ components of each filter by the filter's relative contribution to the mixture. The $r, g, b$ subscripts denote values from Table 1; for example: $X_{r}$ is $22.990, Y_{r}$ is 9.190 , and $\mathrm{X}_{\mathrm{g}}$ is 3.432 .

$$
\begin{aligned}
& X_{m}=p_{r} X_{r}+p_{g} X_{g}+p_{b} X_{b} \\
& Y_{m}=p_{r} Y_{r}+p_{g} Y_{g}+p_{b} Y_{b} \\
& Z_{m}=p_{r} Z_{r}+p_{g} Z_{g}+p_{b} Z_{b}
\end{aligned}
$$

The $x, y$ chromaticity coordinates are calculated from the mixture tristimulus values by the following equations:

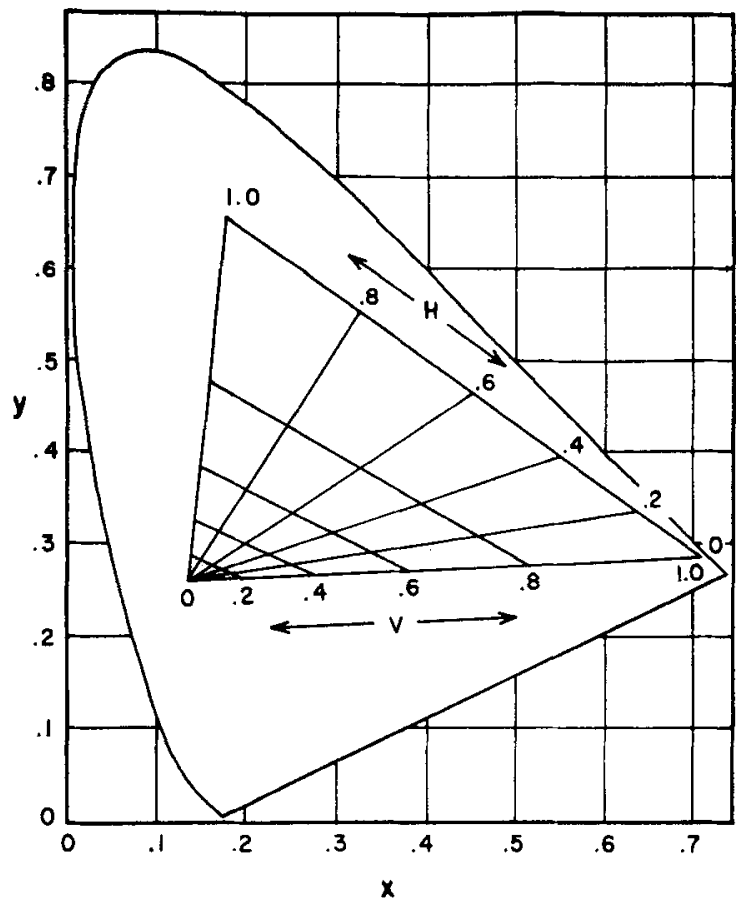

Figure 3. CIE x,y chromaticity diagram. The inscribed triangle represents the maximum possible range of chromaticities with the set of filters described in the text. The points of the triangle are located at the chromaticity coordinates of the filter primaries. The grid lines inside the triangle illustrate how the chromaticity of the mixture varies as a function of the horizontal (H) and vertical (V) position of the filter slide.

$$
\begin{aligned}
& \mathrm{x}=\mathrm{X}_{\mathrm{m}} /\left(\mathrm{X}_{\mathrm{m}}+\mathrm{Y}_{\mathrm{m}}+\mathrm{Z}_{\mathrm{m}}\right) \\
& \mathrm{y}=\mathrm{Y}_{\mathrm{m}} /\left(\mathrm{X}_{\mathrm{m}}+\mathrm{Y}_{\mathrm{m}}+\mathrm{Z}_{\mathrm{m}}\right)
\end{aligned}
$$

The chromaticities obtainable with the arc lamp source and the Wratten filters are circumscribed by the large triangle on the CIE x,y diagram in Figure 3. The grid lines drawn in the triangle represent the translation of the $h, v$ coordinate system into the $x, y$ plane. The overall calibration of the instrument was checked by matching an adjacent field that was illuminated by a lamp of known chromaticity. The standard lamp (General Electric Type 18A/T10/2P$6 \mathrm{~V})$ was operated at a color temperature of $2,800^{\circ} \mathrm{K}$, according to its previous calibration against a Canadian National Research Council standard source (Instrumentation Specialities Company No. 123).

\section{APPLICATIONS}

The first application involved the notoriously difficult matches of colored afterimages (Padgham, 1968). White and Montgomery (Note 2) used the joystick-plotter colorimeter in an experiment designed to measure the effects of memory color on afterimages. Six untrained observers adjusted a colorimeter to match afterimages that resembled either a red, white, and blue flag or a simple striped pattern. The means of six matches by each observer for two of the adaptation targets are shown in Figure 4 on sections of the CIE $x, y$ chromaticity diagram. As the arrows indicate, each observer matched the afterimages of stripes in the flag pattern as redder than afterimages of the same stripes in the striped pattern. The reddish shift for matches involving the memory color occurred with only six matches for each target by untrained observers. No observer reported difficulty with the procedure.

Wolbarsht et al. (Note 1) used a similar colorimeter in an investigation of the effects of various atmospheric conditions on color vision. Since the experimental observations were made in a pressure chamber by observers wearing plastic head tents, the color matching part of the experiment had to be fast and simple. Since the computer was in accessible from the pressure chamber, the joystick control was replaced by two 10-turn potentiometer voltage sources. Observers adjusted the voltages to control the horizontal and vertical position of the filter slide attached to the $\mathrm{X}-\mathrm{Y}$ plotter. The voltages were read from a dual digital voltmeter after a match was completed. We also substituted a 6-V, 9-A ribbon filament lamp for the arc lamp, which reduced the colorimeter's maximum luminance. The targets that were matched were flicker colors produced by rotating a black-and-white pattern on a cylinder in the manner of Benham's top (Festinger et al., 1971). Color 

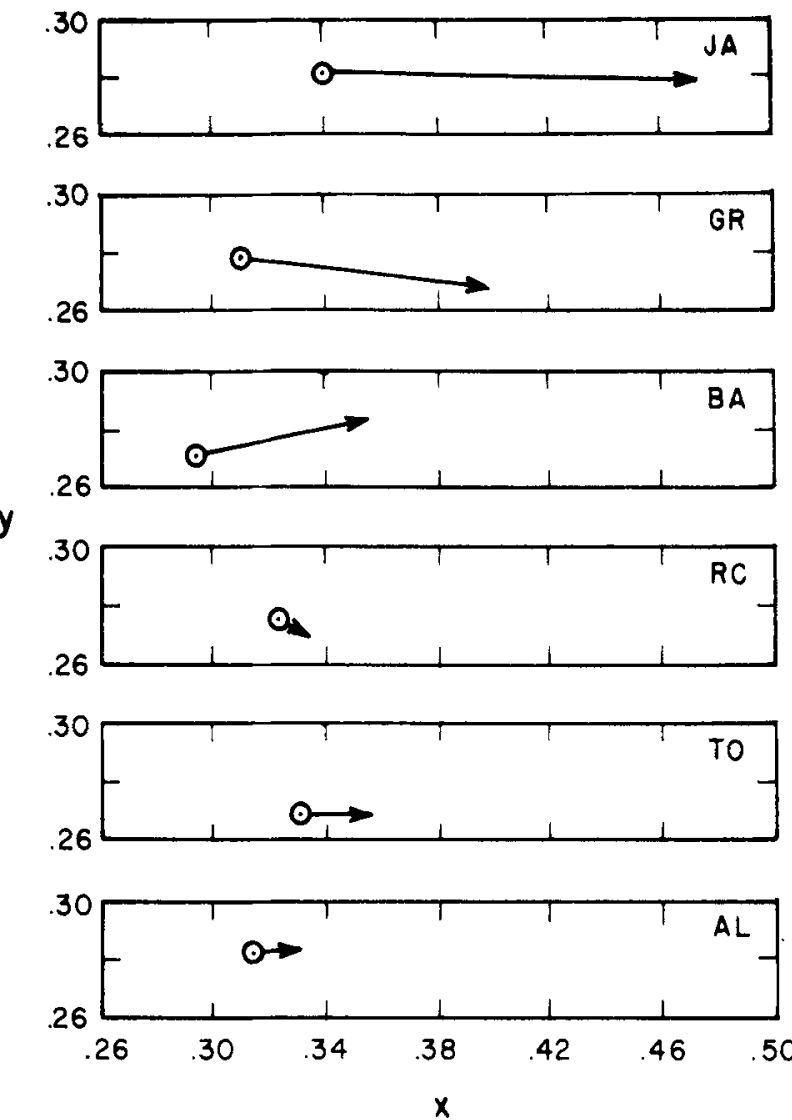

Figure 4. The effect of memory color on afterimages. The separate panels show the afterimage color matches by each of six observers on expanded sections of the CIE $x, y$ diagram. Open circles are matches of afterimages of a pattern of simple stripes. Arrow points indicate matches of stripes in a pattem resembling a negatively colored flag. The chromaticity of both adapting patterns was the same. Each point is the mean of six matches.

matches for three different tlicker patterns at tive simulated altitudes are presented in Figure 5.

\section{VARIATIONS}

The Burnham colorimeter is an extremely flexible instrument. Its range can be moditied to cover a large range of chromaticities by increasing the number of filters on the tilter slide (Burnham, 1952). By adding a blue-green filter such as Wratten No.65. for example, the colorimeter could span a larger portion of the $x, y$ diagram in Figure 3 .

Changing the tilter set can also modify the sensitivity of the colorimeter, which affects the precision of the matches. For example, using less highly saturated primaries reduces the possible range of matches. but increases the precision of matches within the reduced gamul. The joystick-plotter combination makes possible a variable sensitivity colorimeter, since the motion of the hilter slide is controlled by the $X-Y$ plonter input amplitiers.
The utility of the colorimeter might be improved for some applications by adding an intensity control. We have tried manual adjustment of a neutral density wedge between the lens and the filter slide. Although this is relativeiy simple for the observer, the wedge position is not automatically recorded. A better solution would te to add a third-dimension control, perhaps a vertical motion. to the joystick handle to control the wedge position. The additional motion could be described to the observer as moving up and down along the brightness dimension of a color space. If the lilters were judiciously chosen, then the luminance would change very little as the chromaticity was adjusted. Although we did not select filters for equal luminance, the ones we chose did not differ very much. The $Y$ tristimulus values in Table 1 , which are proportional to the luminances, demonstrate that the largest luminance difference occurs between the red and biue tilters. The ratio of the two $Y$ values indicates that the luminance increases only by a factor of about 1.8 . or approximately $0.26 \log _{10}$ units. Such a change occurs only when the filter slide moves from the extreme blue position to the extreme red position. Within the central region of the colorimeter's range, of course. the luminance changes are much less.

The $X-Y$ plotter colorimeter has a unique potential to generate controlled chromaticity stimuli in on-line experiments, if the computer drives the plotter directly through digital-to-analog circuits. This

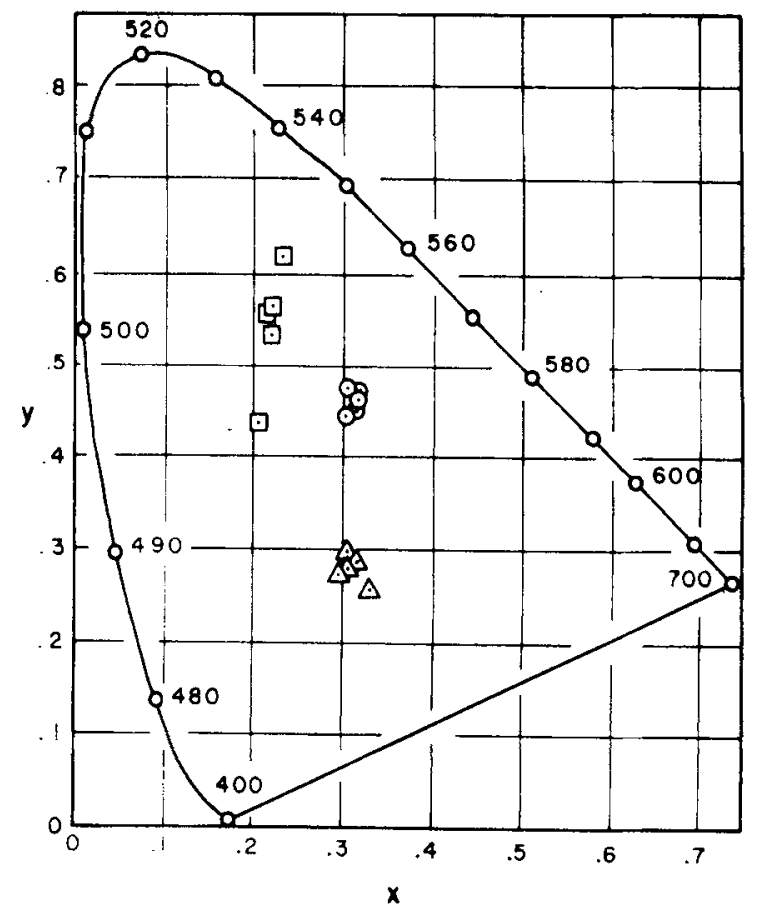

Figure 5. Flicker-color matches. CIE x,y chromaticities for matches of three flicker-color patterns are represented by different shapes. Each set of five points depicts a series of five simulated altitudes from sea level to $30,000 \mathrm{ft}$. Each point is the mean of eight matches by each of five observers. 
operational change has advantages both for presenting stimuli and for recording responses. It makes some new stimuli possible. For example. the computer could rapidly modulate the chromaticity of the stimulus field. or simple signal generators could drive the plotter to modulate the chromaticity. In general. the computer could be programmed to present any sequence of chromaticities to an observer, and the sequence could be contingent on the responses of the observer. Moreover, staircase methods and other psychophysical techniques could be used rather than simple matching procedures in order to control for variability of the observer's criteria.

For classroom demonstrations or for large group experiments, an X-Y plotter could be used with or without the joystick to control the position of the filter slide in a projection colorimeter such as those described by Murch (1972). Murch and Paulson (1974) and Riggs (1964). Due to the small size of the filament image in such a projection device, however, the tolerance for plotter position errors would be much less.

In conclusion, various combinations of joysticks, $X-Y$ plotters, and computers can answer many equipment needs for current research in color vision. In addition, the ease of controlling and manipulating the chromaticity of the colorimeter field suggests new applications for the instrument.

\section{REFERENCE NOTES}

1. Wolbarsht, M. L., White, C. W.. \& Anderson. W. B., Jr. Effect of elimination of nitrogen and/or hypoxiat or restricted visual environment on color vision and range of accommodation N.A.S.A. Fina! Report. Contract NAS 9-11994, 1973.

2. White, C. W., \& Montgomery. D. A. The effect of menory color on afterimages. Presented at the Atlantic Section Meeting of the Association for Research in Vision and Ophthalmology. in Bethesda. Maryland. November 1-2. 1974.

\section{REFERENCES}

Burham, R. W. A colorimeter for research in color perception. Americin Journal of Psychology, 1952, 65, 603-608.

Festinger, L., Allyn, M. R., \& White. C. W. The perception of color with achromatic stimulation. Vision Research, 1971, 11, $591-612$.

Murch, G. M. CIE $x, y$ coordinates from an inexpensive projection colorimeter. Behavior Research Methods \& Instrume'nuation, 1972, 4, 3-5.

Murch. G. M.. \& Paulson, J. An extension of the Riggs projection colorimeter. Behavior Research Methods \& Instrmmentation. 1974. 6. 403-406.

Padgham. C. A. Measurements of the color sequences in positive visual afterinages. Vision Re'search, 1968, 8, 939-949.

Riggs. L. A. A projection color mixer. American Journal of Psychology, 1964, 77, 129-134.

WRIGHT, W. D. The measurement of colour (4th ed.). New York: Van Nostrand Reinhold, 1969.

Wyszecki, G., \& Stiles, W. S. Color science: Concepts and methods, quantitative data and formulas. New York: Wiley, 1967.

(Received for publication November 20.1974; revision received January $25,1975$. ) 